\title{
A Regular $k$-Shrinkage Thresholding Operator for the Removal of Mixed Gaussian-Impulse Noise
}

\author{
Han Pan, Zhongliang Jing, Lingfeng Qiao, and Minzhe Li \\ School of Aeronautics and Astronautics, Shanghai Jiao Tong University, Shanghai, China \\ Correspondence should be addressed to Han Pan; hanpan@sjtu.edu.cn
}

Received 5 April 2017; Accepted 12 June 2017; Published 12 July 2017

Academic Editor: Ridha Ejbali

Copyright (C) 2017 Han Pan et al. This is an open access article distributed under the Creative Commons Attribution License, which permits unrestricted use, distribution, and reproduction in any medium, provided the original work is properly cited.

\begin{abstract}
The removal of mixed Gaussian-impulse noise plays an important role in many areas, such as remote sensing. However, traditional methods may be unaware of promoting the degree of the sparsity adaptively after decomposing into low rank component and sparse component. In this paper, a new problem formulation with regular spectral $k$-support norm and regular $k$-support $\ell_{1}$ norm is proposed. A unified framework is developed to capture the intrinsic sparsity structure of all two components. To address the resulting problem, an efficient minimization scheme within the framework of accelerated proximal gradient is proposed. This scheme is achieved by alternating regular $k$-shrinkage thresholding operator. Experimental comparison with the other state-ofthe-art methods demonstrates the efficacy of the proposed method.
\end{abstract}

\section{Introduction}

Image restoration [1-4] attempts to recover a clear image from the observations of real scenes. As a fundamental procedure, it has been applied to various application areas, such as image fusion [5] and action recognition [6]. However, typically, the noise characteristics of imaging camera is completely or partially unknown. Among these, the removal of mixed noise has not been investigated because the noise model is not easy to establish accurately.

Recently, a patch based method [7] for video restoration has attracted much attention [8-10]. This method also is extended to video in-painting for archived films. However, the mechanisms of modeling the sparsity level of the grouping patches remain unclear.

To deal with the lack of adaptivity in sparsity level [7], a robust video restoration algorithm is proposed. The main idea of the proposed method is to model the sparsity levels of the low rank component by regular spectral $k$-support norm and sparse component by regular $k$-support $\ell_{1}$ norm. Specially, a new problem formulation is presented, where the objective function is minimized under an upper bound constraint on the regularization term. However, it is not easy to solve the resulting problem. Some recent progress [11] in the theory of optimization on iterative shrinkage thresholding method is considered. And, an efficient alternating minimization scheme is proposed to solve the new objective.

1.1. Related Works. Recently, the problem of denoising image corrupted by mixed Gaussian-impulse noise has been studied in many different contexts [8-10, 12, 13]. These methods fall into three categories: variational methods [9, 12], sparse representation [8, 10], and patch based method [7].

Variational methods are a new class of the solutions to promote edge-preservation, such as total variation [14]. These methods first utilized some spatial filters to detect and remove the corrupted pixels, for example, adaptive center-weighted median filter [15] (ACWMF) or rank order absolute differences [16] (ROAD) detector. In [12], Cai et al. employed Mumford-Shah regularization term to encourage sparsity in gradient domain. In [9], Rodríguez et al. presented a novel optimization method for the generalized total variation regularization method. It can be seen that the denoised performance of these methods relies on the detection for the damaged candidates. The adaptivity of sparsity level of the regularization terms has not been investigated carefully.

Sparse representation based methods have been extended to this problem. In the main idea of this scheme, it is assumed 
that the signal can be described by linear combination of a spare number of elements or atoms of an overcomplete dictionary. In [8], an efficient image reconstruction method by posing $\ell_{1}$ norm on the error, and $\ell_{0}$ norm on image patches in learned dictionary, was proposed. In [10], Filipovic and Jukic reformulated a new problem formulation by enforcing $\ell_{0}-\ell_{1}$ sparsity constraints. The resulting problem is solved by a mixed soft-hard thresholding method. However, it should be noted that these methods are time-consuming.

Patch based method is proven to be a state-of-the-art denoising scheme. In [7], Ji et al. approximated the patch stack by reformulating the problem as a low rank matrix completion problem. Despite its efficacy, one of the limitations of patch based method in [7] is that the degree of sparsity has not been considered carefully. When the underlying sparsity level is unknown, we may obtain a bias estimate, considerably. To alleviate these issues in a unified formulation, a new problem formulation is proposed.

1.2. Contributions. The main idea of this paper is to deal with the weakness of the approach in [7]. Existing methods, such as $\ell_{1}$ norm and trace norm, can not promote the sparsity level of all two components adaptively. The details or local fine content can not be represented and described well. To deal with these issues, a new problem formulation incorporating correlated and adaptive sparsity is proposed.

Our contributions can be summarized as follows.

(1) A new problem formulation to model the sparsity level of the patches is proposed. A new norm extended from $k$-support norm and ordered $\ell_{1}$ norm is presented.

(2) An efficient minimization scheme with regular $k$ shrinkage thresholding operator is proposed, which is based on the optimization framework of accelerated proximal gradient (APG) method.

(3) Numerical experiments, compared to other stateof-the-art methods, demonstrate that the proposed method outperforms the related restoration methods.

1.3. Organization. The remainder of this paper is presented as follows. In Section 2, some basic notations are provided. In Section 3, a detailed description about the proposed objective function is given. In Section 4, an efficient minimization scheme within the framework of APG is proposed. Then, some experiments are conducted to validate the effectiveness of the proposed method in Section 5. Finally, we conclude the paper in Section 6.

\section{Preliminaries}

There are some notations presented for the simplicity of discussions. Frobenius norm and $\ell_{1}$ norm of a matrix $X \in$ $\mathbb{R}^{m \times n}$ are defined by $\|X\|_{F}$ and $\|X\|_{1}$, respectively. For a scalar $\tau$, the shrinkage operator [17] $S_{\tau}(x)$ for $\ell_{1}$ norm minimization problem is defined as follows:

$$
S_{\tau}(x)=\operatorname{sgn}(x) \max (|x|-\tau, 0),
$$

where sgn is a signum function; $|\cdot|$ calculates the absolute value.

Assuming that $X$ is of rank $r$, the singular value decomposition (SVD) of $X$ with nonnegative singular values is defined by $X=U \Sigma V^{T}$, where $\Sigma$ denotes a diagonal matrix with the singular values. Based on the SVD computation, the nuclear norm is defined in the following way:

$$
\|X\|_{*}=\operatorname{tr}\left(\sqrt{X^{t} X}\right)=\operatorname{tr}\left(\sqrt{V \Sigma^{2} V^{T}}\right)=\sum_{i=1}^{r}\left|\sigma_{i}\right|,
$$

where $\sigma_{i}$ is the $i$ th largest singular value of $X$. A solution with shrinkage operator to the nuclear norm is singular value shrinkage operator [18] $D_{\tau}(X)$, which can be expressed as follows:

$$
D_{\tau}(X)=U \Sigma_{\tau} V^{T},
$$

where $\Sigma_{\tau}: \mathbb{R} \rightarrow \mathbb{R}$ is defined as follows:

$$
\Sigma_{\tau}=\operatorname{diag}\left(\operatorname{sgn}\left(\sigma_{i}\right) \max \left(\left|\sigma_{i}\right|-\tau, 0\right)\right) .
$$

However, it should be noted that the shrinkage operator for $\ell_{1}$ norm is different from singular value shrinkage operator for nuclear norm. There operators play an important role in joint sparse and low rank matrix approximation.

In this paper, ordered $\ell_{1}$-norm [19] is provided as follows:

$$
\ell_{\mathrm{O} 1}=\sum_{i=1}^{n} w|x|_{i}
$$

where $x$ is sorted in decreasing order. $|x|_{i}$ denotes the $i$ th largest element of the magnitude vector $|x|=$ $\left(\left|x_{1}\right|, \ldots,\left|x_{n}\right|\right)^{T} . w$ is a trade-off vector in nonincreasing order. When $w$ is a constant vector, (5) reduces to $\ell_{1}$ norm. When $w_{1}>0$ and $w_{2 \leq i \leq n}=0$, then (5) reduces to $\ell_{\infty}$-norm.

$k$-support norm [20] is defined as follows:

$$
k_{s}(x)=\left\{x \in \mathbb{R}^{n}:\|x\|_{0} \leq S,\|x\|_{2} \leq 1\right\},
$$

where $S$ denotes the bound of the sparsity level of $x$, which is a positive integer. The details of $k$-support norm are in traduced in [20,21]. It has been extended to the case of matrix, which named by regular spectral $k$-support norm. Although this norm provides the number of elements of the sparsity level, it lacks of efficient mechanism to promote the sparsity adaptively.

After taking advantage of both ordered $\ell_{1}$ norm and $k$-support norm, regular $k$-support $\ell_{1}$ norm is defined as follows:

$$
\lambda\|x\|_{1, k}=\sum_{i=1}^{k} \lambda_{i}|x|_{i},
$$

where $\lambda$ is a positive regularization vector in nonincreasing order. And $1 \leq k \leq n$, where $n$ is the size of vector $x$.

For the case of matrix, regular spectral $k$-support norm is proposed, which can be expressed as follows:

$$
\lambda\|X\|_{k}^{*}=\sum_{i=1}^{k} \lambda_{i}\left|\sigma_{i}\right|, \quad X \in \mathbb{R}^{m \times n},
$$


where $1 \leq k \leq \min (m, n)$. It can be noted that the singular values are arranged in nonincreasing order.

\section{Problem Setup}

This section introduces the objective function in detail. For each reference patch $p$, similar patches in the spatialand temporal-domain are obtained by utilizing the patch matching algorithm. The matched patches are denoted as $\left\{p_{i}\right\}_{i=1}^{m}$, where $m$ stands for the size. It can be noted that each patch $p_{i}$ is rearranged as a vector with size $\mathbb{R}^{n^{2}}$ through concatenating all columns into a column vector. At last, a matrix $O \in n^{2} \times m$ is generated after considering all the $m$ patches, which can be represented as follows:

$$
O=\left(p_{1}, p_{2}, \ldots, p_{m}\right)
$$

In this paper, we assumed that the observed patch matrix $O$ can be decomposed into three components:

$$
O=L+S+N
$$

where $L$ stands for low rank component, $S$ is sparse component, and $N$ is additive noise. There are some regularization methods for (10), such as $L$ with nuclear norm (also known as the trace norm) and $S$ with $\ell_{1}$ norm. The problem formulation in [7] can be expressed as follows:

$$
\begin{array}{ll}
\min _{L, S} & \|L\|_{*}+\lambda\|S\|_{1}, \\
\text { s.t. } & \|O-L-S\|_{F} \leq \epsilon,
\end{array}
$$

where $\|\cdot\|_{*}$ is nuclear norm, and $\|\cdot\|_{1}$ for $\ell_{1}$ norm.

However, these norms may lead to a large estimation bias [19] but can not promote the sparsity level adaptively. For example, the limitations of $\ell_{1}$ norm have been investigated in $[22,23]$. Similarly, some alternative cases, such as the $\ell_{p}$ quasinorm, also have been discussed in [24-26]. Thus, a suitable solution is required to recover these components.

To alleviate these limitations, a new problem formulation is proposed to model the sparsity levels both on $L$ and $S$. Moreover, a unified formulation to describe the correlated variables is considered. To estimate the underlying structures of $L$ and $S$, we focus on the following minimization function:

$$
\begin{array}{ll}
\min _{L, S} & \lambda_{1}\|L\|_{k}^{*}+\lambda_{2}\|S\|_{1, k}, \\
\text { s.t. } & \|O-L-S\|_{F} \leq \epsilon,
\end{array}
$$

where $\lambda_{1}$ and $\lambda_{2}$ are two positive regularization vectors in a nondecreasing order. $\lambda_{1}\|L\|_{k}^{*}$ stands for regular spectral $k$ support norm on $L$. $\lambda_{2}\|S\|_{1, k}$ denotes regular $k$-support $\ell_{1}$ norm on $S$. And $\epsilon$ is the standard deviation of noise $N$.

The above formulation amounts to the constraint $\| O-$ $L-S \|_{F} \leq \epsilon$, which is considered more natural than usual formulation because it stands for the tolerance on the error.
After choosing a suitable $\epsilon$, (12) can be reformulated as follows:

$$
\min _{L, S} \lambda_{1}\|L\|_{k}^{*}+\lambda_{2}\|S\|_{1, k}+\frac{1}{2 \mu}\|O-L-S\|_{F}^{2},
$$

where $\mu$ is a suitable positive value. It can be seen that these are some challenges to solve (13). First, regular $k$-support $\ell_{1}$-norm is posed on sparse component $S$. Second, the low rank component $L$ is penalized by regular spectral $k$-support norm.

There are several properties of our problem formulation in (13). First, the proposed regular spectral $k$-support norm on $L$ and regular $k$-support $\ell_{1}$ norm on $S$ aim to reconstruct the local structures clearly. It should be noted that these modeling strategies can adaptively promote the sparsity level with an upper bound. Second, to the best of our knowledge, this is the first time of combining the advantages of both ordered $\ell_{1}$ norm and $k$-support norm to yield a robust subspaces estimation against noise. Third, although the optimization method in [27] is very similar to the proposed method, the proposed method can deal with more complex situations. Moreover, the proposed method can adopt the more challenging situations, such as the removal of mixed Gaussian, salt-and-pepper noise, and random value impulse noise. It should be noted that this noisy situation has not been explored in [7].

\section{Proposed Method}

4.1. Proposed Framework. In this section, an optimization framework using regular $k$-shrinkage thresholding operator is presented. First, accelerated proximal gradient method (APG) is applied to the resulting problem because of its simplicity and popularity in imaging applications $[28,29]$. Second, the proposed regular $k$-shrinkage thresholding operator is applied to the two resulting subproblems. As showed in $[22,23]$, nonconvex regularization functions have been shown both theoretically and experimentally to provide better results than $\ell_{1}$ norm. Then, some explicit proximal mappings are developed.

APG based scheme aims to solve an unconstrained minimization problem by

$$
\min _{X} g(X)+f(X)
$$

where $g$ is assumed to be a nonsmooth function and $f$ for a smooth function. Here, $L_{f}$ denotes the Lipschitz constant of the gradient of $f$.

Applying the framework of APG to problem (13), we have the following expressions:

$$
\begin{aligned}
X & =(S, L), \\
g(X) & =\mu \lambda_{1}\|L\|_{k}^{*}+\mu \lambda_{2}\|S\|_{1, k}, \\
f(X) & =\frac{1}{2}\|O-L-S\|_{F}^{2} .
\end{aligned}
$$




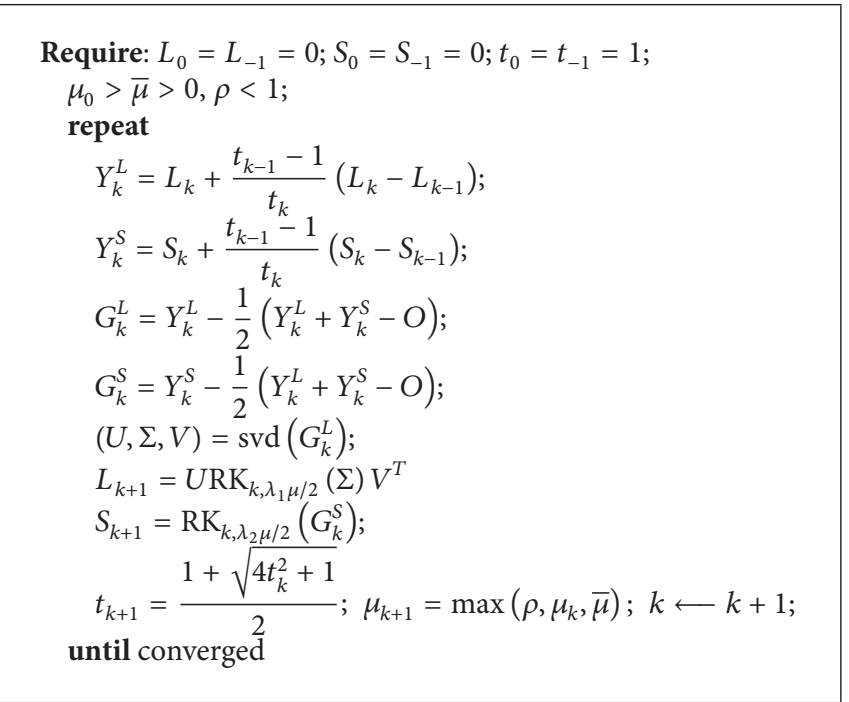

Algorithm 1: An efficient minimization scheme with regular $k$-shrinkage thresholding operator.

Setting $L_{f}=1$, then we have the following objective function:

$$
\begin{aligned}
\min _{L, S} & \mu \lambda_{1}\|L\|_{k}^{*}+\mu \lambda_{2}\|S\|_{1, k}+\left\|L-G_{k}^{L}\right\|_{F}^{2} \\
& +\left\|S-G_{k}^{S}\right\|_{F}^{2},
\end{aligned}
$$

where the proximal points $G_{k}^{L}$ and $G_{k}^{S}$ in the framework of APG are defined in Algorithm 1. It can be noted that both the variables $L$ and $S$ are separable. Thus, there are two subproblems, which can be represented as follows:

$$
\begin{array}{ll}
\min _{L} & \mu \lambda_{1}\|L\|_{k}^{*}+\left\|L-G_{k}^{L}\right\|_{F}^{2}, \\
\min _{S} & \mu \lambda_{2}\|S\|_{1, k}+\left\|S-G_{k}^{S}\right\|_{F}^{2} .
\end{array}
$$

It can be seen that (17) is a minimization problem with regular spectral $k$-support norm and (18) with regular $k$-support $\ell_{1}$-norm. To deal with these problems, regular $k$-shrinkage thresholding operator (RK) is defined as follows:

$$
\mathrm{RK}_{k, \tau}(x)=\operatorname{sgn}(x) \max (|x| \ominus \tau, 0), \quad\|x\|_{0} \leq k,
$$

where $\ominus$ denotes an operation of direct minus in nonincreasing order. $k$ denotes the sparsity level of the input vector. $\tau$ also is a vector in a nonincreasing order.

Remark 1. There are some differences between regular $k$ shrinkage thresholding operator and shrinkage operator [17]. First, the proposed operator models the sparsity level by the procedure of regular shrinkage adaptively. Second, the introduction of $k$-support constraint can bound the degree of the sparsity. Third, the combination of regular shrinkage and $k$-support leads to the modeling of the correlated variables robustly. have

When applying the proposed operator to $G_{k}^{L}$ and $G_{k}^{S}$, we

$$
\begin{aligned}
& L_{k+1}=\operatorname{URK}_{k, \lambda_{1} \mu / 2}(\Sigma) V^{T}, \\
& S_{k+1}=\operatorname{RK}_{k, \lambda_{2} \mu / 2}\left(G_{k}^{S}\right),
\end{aligned}
$$

where $\Sigma$ denotes the eigenvalues of $G_{k}^{L}$. It should be noted that the solution to $L_{k+1}$ can be viewed as a generalization of singular value shrinkage operator. Based on the framework of APG, an optimization framework for the objective function (16) is presented in Algorithm 1. The detailed procedures for the two subproblems are provided in Algorithm 1.

4.2. Some Implementation Details. In our implementation, the sampled image patches with overlapping regions are considered. Then, each frame of the restored video may be replaced by the recovered patches. For the synthesis process, the outcome of each selected pixel is accomplished by calculating the average of multiple estimates from the related patches. This procedure could deal with the artifacts along the boundaries of patches and restore fine details locally.

\section{Experiments and Discussion}

5.1. Experimental Settings. To demonstrate the effectiveness and efficacy of the proposed method, some experiments are conducted. We focus on the removal of mixed Gaussianimpulse noise. Two types of noisy situations, including mixed Gaussian and random value impulse noise (GRV) denoted by $(\sigma, s i)$ and mixed Gaussian, salt-and-pepper noise, and random value impulse noise (GSPRV) by ( $\sigma$, sp, si), are tested. Some samples of three videos (http://trace.eas.asu.edu/yuv/) are displayed in Figure 1. The sizes of coastguard, flower, and news in our experiments are $176 \times 144 \times 100,352 \times 240 \times 150$, and $352 \times 288 \times 150$, respectively. The parameter $k$ of the 


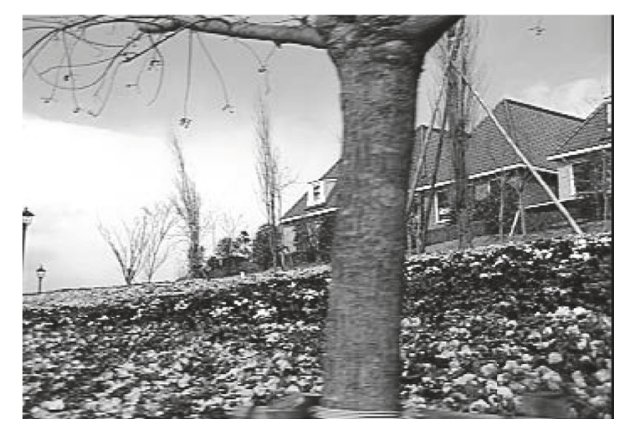

(a)

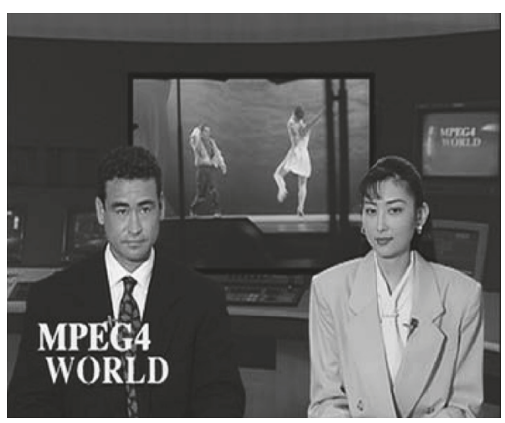

(b)

Figure 1: Two samples used in the experiments ((a) sample for flower, (b) sample for news).

TABLE 1: Numerical results by the removal of MGRV, measured by PSNR and FSIM.

\begin{tabular}{|c|c|c|c|c|c|}
\hline Noise level & Indexes & Methods & Coastguard & Flower & News \\
\hline \multirow{4}{*}{$(10,10 \%)$} & \multirow{4}{*}{ PSNR } & VBM3D & 28.75 & 19.62 & 30.36 \\
\hline & & RPCA & 30.75 & 22.11 & 33.49 \\
\hline & & $\ell_{1}-\ell_{0}$ & 28.49 & 19.45 & 28.40 \\
\hline & & Ours & 31.07 & 23.01 & 34.86 \\
\hline \multirow{4}{*}{$(15,20 \%)$} & \multirow{4}{*}{ PSNR } & VBM3D & 27.37 & 18.72 & 28.10 \\
\hline & & RPCA & 28.98 & 19.66 & 30.24 \\
\hline & & $\ell_{1}-\ell_{0}$ & 26.15 & 18.12 & 25.56 \\
\hline & & Ours & 29.58 & 21.41 & 31.32 \\
\hline \multirow{4}{*}{$(20,30 \%)$} & \multirow{4}{*}{ PSNR } & VBM3D & 25.56 & 17.97 & 25.64 \\
\hline & & RPCA & 25.69 & 18.23 & 26.34 \\
\hline & & $\ell_{1}-\ell_{0}$ & 23.06 & 16.66 & 24.20 \\
\hline & & Ours & 27.17 & 19.30 & 27.41 \\
\hline \multirow{4}{*}{$(10,10 \%)$} & \multirow{4}{*}{ FSIM (\%) } & VBM3D & 87.71 & 81.64 & 95.52 \\
\hline & & RPCA & 90.01 & 87.57 & 96.47 \\
\hline & & $\ell_{1}-\ell_{0}$ & 91.35 & 84.94 & 90.11 \\
\hline & & Ours & 92.04 & 92.14 & 96.35 \\
\hline \multirow{4}{*}{$(15,20 \%)$} & \multirow{4}{*}{ FSIM (\%) } & VBM3D & 82.24 & 75.90 & 92.74 \\
\hline & & RPCA & 89.32 & 79.93 & 93.47 \\
\hline & & $\ell_{1}-\ell_{0}$ & 84.33 & 79.25 & 87.10 \\
\hline & & Ours & 90.13 & 87.58 & 94.06 \\
\hline \multirow{4}{*}{$(20,30 \%)$} & \multirow{4}{*}{ FSIM (\%) } & VBM3D & 77.19 & 70.42 & 89.18 \\
\hline & & RPCA & 86.69 & 74.30 & 88.35 \\
\hline & & $\ell_{1}-\ell_{0}$ & 79.47 & 73.35 & 82.41 \\
\hline & & Ours & 87.82 & 77.09 & 89.48 \\
\hline
\end{tabular}

proposed method for the coastguard is set to 1000. For the other videos, that is, flower and news, $k$ is set to 2000. All experiments are performed in MATLAB R2014 running on a desktop with Intel Core i7 at $3.2 \mathrm{GHz}$.

Three related methods are compared with the proposed method, including VBM3D [30], RPCA based method [7], and $\ell_{0}-\ell_{1}$ based method [8]. VBM3D based method is not originally designed for the removal of mixed Gaussianimpulse noise. To remedy this problem, adaptive centerweighted median filter [15] (ACWMF) is used to detect and remove the impulse noise firstly. Two indexes are taken for assessing the denoised performance of all competing methods, that is, peak-signal-to-noise ratio (PSNR) and feature-similarity (FSIM) index [31].

5.2. Mixed Gaussian and Random Value Impulse Noise. In this subsection, the denoising results for three different scenarios are presented, including $(\sigma, \mathrm{si})=(10,10 \%),(15,20 \%)$, and $(20,30 \%)$. Numerical results on three videos are presented in Table 1. It can be observed that the proposed 


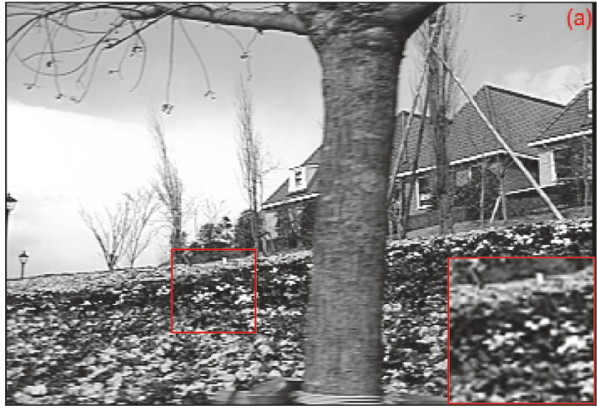

(a)

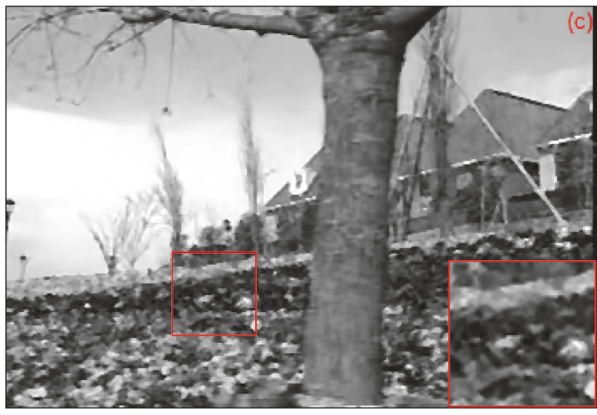

(c)

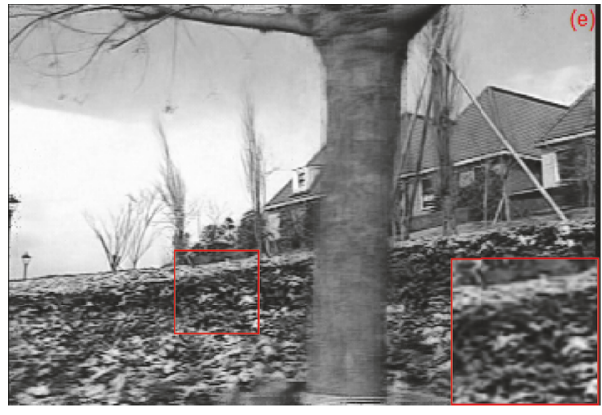

(e)

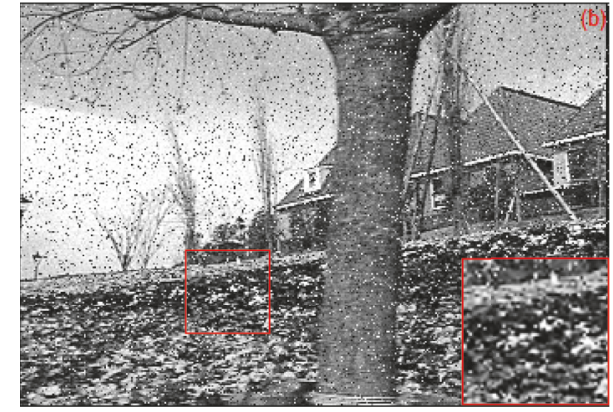

(b)

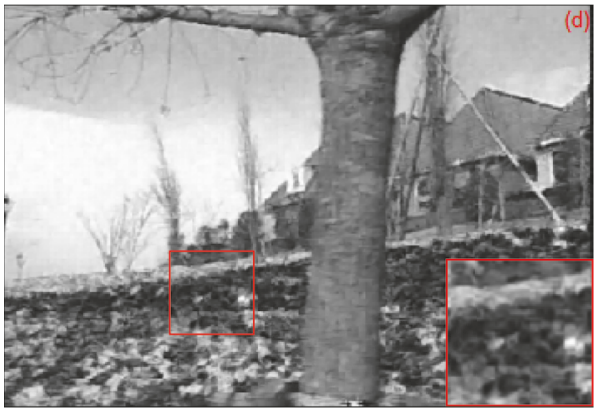

(d)

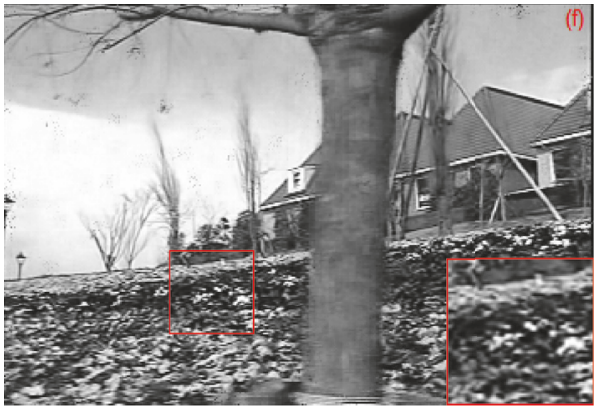

(f)

FIGURE 2: Visual comparison of denoising results on flower by noise level $(\sigma, \mathrm{si})=(10,10 \%)$. (a) Original sample (flower), (b) noisy image, (c) VBM3D based method (PSNR = 19.62), (d) RPCA based method (PSNR = 22.11), (e) $\ell_{1}-\ell_{0}$ based method $($ PSNR $=19.45)$, and $(\mathrm{f})$ the proposed method $(\mathrm{PSNR}=23.01, k=2000)$.

method outperforms all the competing methods with respect to FSIM and PSNR. Visual outcomes are demonstrated in Figure 2. The recovered result of proposed method is presented in Figure 2(f). To examine the recovered details, the selected parts in the visual results are enlarged. It can be noted that the proposed method can reconstruct more local details.

\subsection{Mixed Gaussian, Salt-and-Pepper Noise, and Random} Value Impulse Noise. In this subsection, the experimental results by the removal of Gaussian, salt-and-pepper, and random value impulse noise are demonstrated. Two noisy levels are assessed. The numerical results are presented in Table 2. It can be noted that the proposed method outperforms other methods. A visual assessment of the reconstruction performance of both algorithms is shown in Figure 3. As shown in the enlarged parts, the proposed method presented in Figure 3(f) recovers more local details than other methods.

5.4. Discussion. In this paper, an efficient image restoration scheme for hybrid Gaussian-impulse noise is proposed. The denoising performance of our method is examined in various noisy scenarios. When the strength of noisy levels increased, our method performed more efficiently than other methods. The outcomes of all experiments verified the effectiveness of the proposed method. The difference may be related to the modeling method and optimization strategy we taken. Moreover, the intrinsic sparsity structure of each decomposition component is explored. It should be noted that some limitations may be observed, such as being oversmooth on the local region.

In this paper, an alternating minimization method with regular $k$-shrinkage thresholding operator is proposed. 


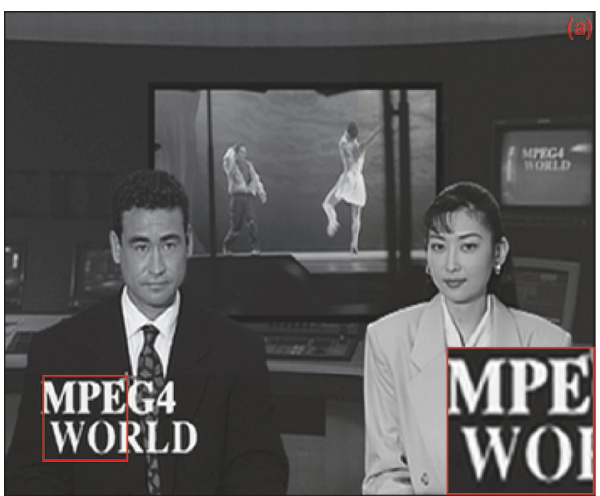

(a)

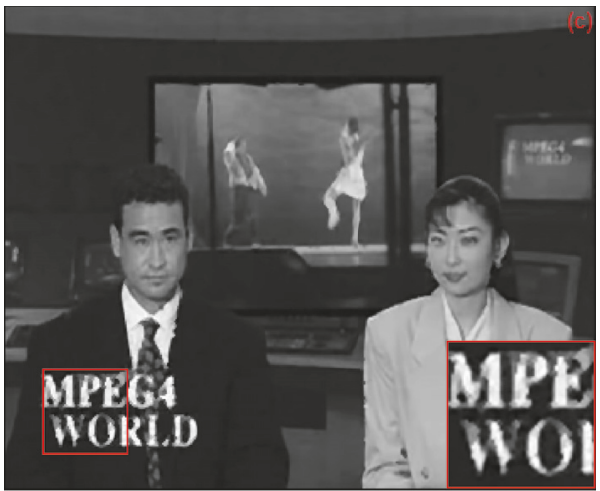

(c)

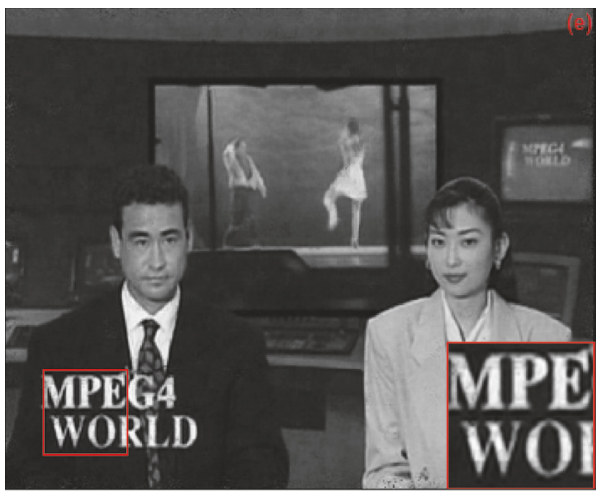

(e)

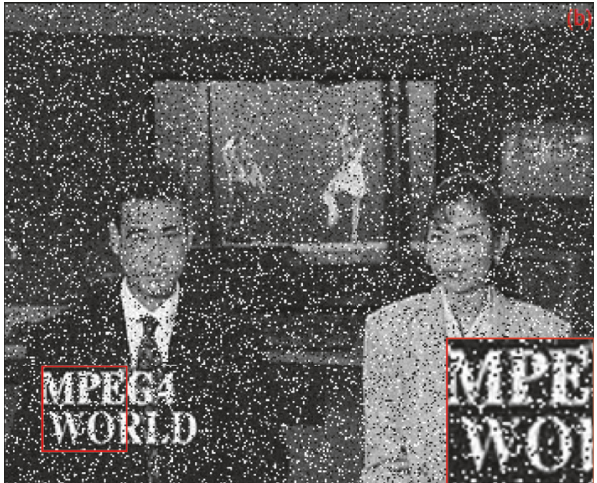

(b)

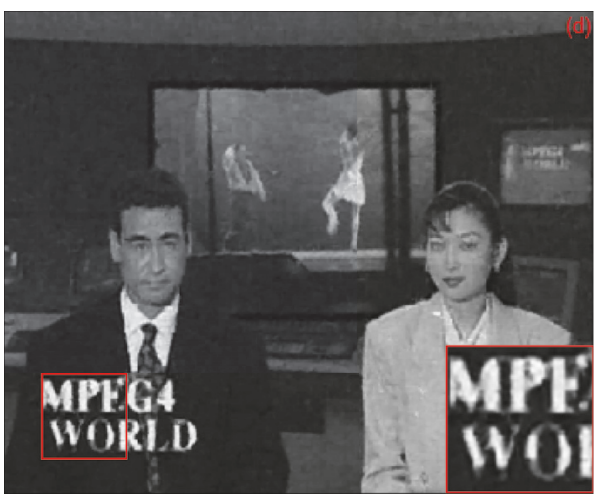

(d)

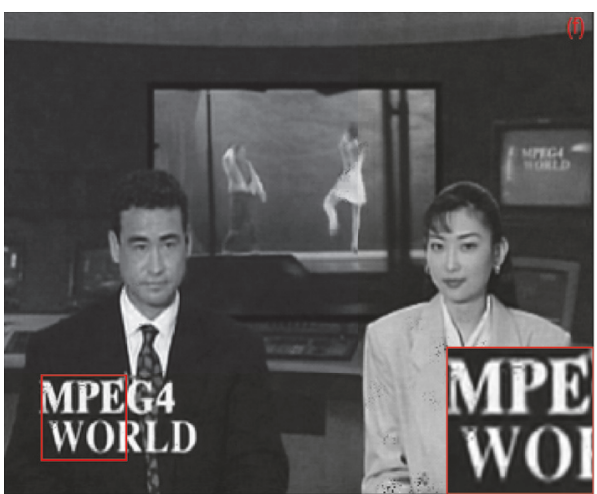

(f)

FIGURE 3: Visual comparison of denoising results by noise level (15, 15\%, 15\%). (a) Original sample (news), (b) noisy image, (c) VBM3D based method (PSNR $=26.23)$, (d) RPCA based method (PSNR $=24.60),(\mathrm{e}) \ell_{1}-\ell_{0}$ based method (PSNR = 24.87), and (f) the proposed method $(\mathrm{PSNR}=28.01, k=2000)$.

Specially, a universal modeling strategy by exploiting the adaptivity of sparsity structure leads to higher quality reconstructions. The proposed method may provide a new class of denoising methods to deal with mixed Gaussian-impulse noise. The numerical results from various experiments validated the effectiveness of the proposed method again.

\section{Conclusion}

In this paper, an efficient video restoration scheme is proposed for the removal of mixed Gaussian-impulse noise.
Unlike traditional $\ell_{1}$ norm based methods, which treat all the values equally, the proposed method tries to explore the additional structure by regular spectral $k$-support norm on low rank component and regular $k$-support $\ell_{1}$ norm on sparse component. Then, the special structure can be promoted on the sparsity level of the decomposition matrices adaptively. To overcome the nonconvex problem, a solution with alternating regular $k$-shrinkage thresholding operator is proposed. The proposed method has good practical performance with appropriate structures. The numerical results, compared to 
TABLE 2: Numerical results by the removal of GSPRV, measured by PSNR and FSIM.

\begin{tabular}{|c|c|c|c|c|c|}
\hline Noise level & Indexes & Methods & Coastguard & Flower & News \\
\hline \multirow{4}{*}{$(10,10 \%, 10 \%)$} & \multirow{4}{*}{ PSNR } & VBM3D & 24.54 & 18.13 & 27.22 \\
\hline & & RPCA & 24.72 & 18.98 & 28.40 \\
\hline & & $\ell_{1}-\ell_{0}$ & 26.27 & 18.35 & 27.05 \\
\hline & & Ours & 25.27 & 20.51 & 29.32 \\
\hline \multirow{4}{*}{$(15,15 \%, 15 \%)$} & \multirow{4}{*}{ PSNR } & VBM3D & 23.79 & 17.62 & 26.23 \\
\hline & & RPCA & 22.11 & 16.65 & 24.60 \\
\hline & & $\ell_{1}-\ell_{0}$ & 24.21 & 17.37 & 24.87 \\
\hline & & Ours & 24.26 & 18.67 & 28.01 \\
\hline \multirow{4}{*}{$(10,10 \%, 10 \%)$} & \multirow{4}{*}{ FSIM (\%) } & VBM3D & 85.32 & 78.72 & 93.52 \\
\hline & & RPCA & 89.50 & 82.94 & 93.75 \\
\hline & & $\ell_{1}-\ell_{0}$ & 88.62 & 79.44 & 90.66 \\
\hline & & Ours & 89.43 & 87.64 & 94.13 \\
\hline \multirow{4}{*}{$(15,15 \%, 15 \%)$} & \multirow{4}{*}{ FSIM (\%) } & VBM3D & 80.05 & 74.39 & 90.87 \\
\hline & & RPCA & 83.33 & 75.13 & 82.78 \\
\hline & & $\ell_{1}-\ell_{0}$ & 83.57 & 75.16 & 84.61 \\
\hline & & Ours & 87.72 & 79.92 & 91.83 \\
\hline
\end{tabular}

some state-of-the-art methods, demonstrate the advantages of the proposed method.

\section{Conflicts of Interest}

The authors declare that they have no conflicts of interest.

\section{Acknowledgments}

This work is jointly supported by National Natural Science Foundation of China (Grants nos. 61603249 and 61673262) and key project of Science and Technology Commission of Shanghai Municipality (Grant no. 16JC1401100).

\section{References}

[1] R. Das, S. Thepade, S. Bhattacharya, and S. Ghosh, "Retrieval architecture with classified query for content based image recognition," Applied Computational Intelligence and Soft Computing, vol. 2016, Article ID 1861247, 9 pages, 2016.

[2] H. Pan, Z. Jing, M. Lei, R. Liu, B. Jin, and C. Zhang, "A sparse proximal Newton splitting method for constrained image deblurring," Neurocomputing, vol. 122, pp. 245-257, 2013.

[3] M. Kumar, S. K. Mishra, and S. S. Sahu, "Cat swarm optimization based functional link artificial neural network filter for gaussian noise removal from computed tomography images," Applied Computational Intelligence and Soft Computing, vol. 2016, Article ID 6304915, 6 pages, 2016.

[4] G. Zhang, P. Jiang, K. Matsumoto, M. Yoshida, and K. Kita, "Reidentification of persons using clothing features in real-life video," Applied Computational Intelligence and Soft Computing, vol. 2017, Article ID 5834846, 9 pages, 2017.

[5] H. Pan, Z. Jing, R. Liu, and B. Jin, "Simultaneous spatial-temporal image fusion using Kalman filtered compressed sensing," Optical Engineering, vol. 51, no. 5, pp. 23-29, 2012.

[6] X. Li, Y. Zhang, and D. Liao, "Mining key skeleton poses with latent svm for action recognition," Applied Computational Intelligence and Soft Computing, vol. 2017, Article ID 5861435, 11 pages, 2017.
[7] H. Ji, S. Huang, Z. Shen, and Y. Xu, "Robust video restoration by joint sparse and low rank matrix approximation," SIAM Journal on Imaging Sciences, vol. 4, no. 4, pp. 1122-1142, 2011.

[8] Y. Xiao, T. Zeng, J. Yu, and M. K. Ng, "Restoration of images corrupted by mixed Gaussian-impulse noise via 11-10 minimization," Pattern Recognition, vol. 44, no. 8, pp. 1708-1720, 2011.

[9] P. Rodríguez, R. Rojas, and B. Wohlberg, "MIxed Gaussianimpulse noise image restoration via total variation," in Proceedings of the 2012 IEEE International Conference on Acoustics, Speech, and Signal Processing, ICASSP 2012, pp. 1077-1080, March 2012.

[10] M. Filipovic and A. Jukic, "Restoration of images corrupted by mixed gaussian-impulse noise by iterative soft-hard thresholding," in Proceedings of the 22nd European Signal Processing Conference (EUSIPCO), pp. 1637-1641, IEEE, 2014.

[11] J. Woodworth and R. Chartrand, "Compressed sensing recovery via nonconvex shrinkage penalties," Mathematics, vol. 2012, no. 7, article 075004, 2015.

[12] J.-F. Cai, R. H. Chan, and M. Nikolova, "Two-phase approach for deblurring images corrupted by impulse plus Gaussian noise," Inverse Problems and Imaging, vol. 2, no. 2, pp. 187-204, 2008.

[13] Y.-R. Li, L. Shen, D.-Q. Dai, and B. W. Suter, "Framelet algorithms for de-blurring images corrupted by impulse plus Gaussian noise," IEEE Transactions on Image Processing, vol. 20, no. 7, pp. 1822-1837, 2011.

[14] L. I. Rudin, S. Osher, and E. Fatemi, "Nonlinear total variation based noise removal algorithms," Physica D. Nonlinear Phenomena, vol. 60, no. 1-4, pp. 259-268, 1992.

[15] T. Chen and H. R. Wu, "Adaptive impulse detection using center-weighted median filters," IEEE Signal Processing Letters, vol. 8, no. 1, pp. 1-3, 2001.

[16] R. Garnett, T. Huegerich, C. Chui, and W. He, "A universal noise removal algorithm with an impulse detector," IEEE Transactions on Image Processing, vol. 14, no. 11, pp. 1747-1754, 2005.

[17] D. L. Donoho, "De-noising by soft-thresholding," IEEE Transactions on Information Theory, vol. 41, no. 3, pp. 613-627, 1995. 
[18] J.-F. Cai, E. J. Candès, and Z. Shen, "A singular value thresholding algorithm for matrix completion," SIAM Journal on Optimization, vol. 20, no. 4, pp. 1956-1982, 2010.

[19] M. Bogdan, E. V. D. Berg, W. Su, and E. Candes, "Statistical estimation and testing via the sorted 11 norm," Statistics, 2013.

[20] A. Argyriou, R. Foygel, and N. Srebro, "Sparse prediction with the $k$-support norm," Advances in Neural Information Processing Systems, vol. 2, pp. 1466-1474, 2012.

[21] A. Eriksson, T. T. Pham, T.-J. Chin, and I. Reid, "The ksupport norm and convex envelopes of cardinality and rank," in Proceedings of the IEEE Conference on Computer Vision and Pattern Recognition, CVPR 2015, pp. 3349-3357, June 2015.

[22] R. Gribonval and M. Nielsen, "Sparse representations in unions of bases," IEEE Transactions on Information Theory, vol. 49, no. 12, pp. 3320-3325, 2003.

[23] A. M. Bruckstein, D. L. Donoho, and M. Elad, "From sparse solutions of systems of equations to sparse modeling of signals and images," SIAM Review, vol. 51, no. 1, pp. 34-81, 2009.

[24] R. Saab, R. Chartrand, and Ö. Yilmaz, "Stable sparse approximations via nonconvex optimization," in Proceedings of the IEEE International Conference on Acoustics, Speech and Signal Processing (ICASSP '08), pp. 3885-3888, April 2008.

[25] X. Chen, F. Xu, and Y. Ye, "Lower bound theory of nonzero entries in solutions of ell $_{2}$-ell p minimization," SIAM Journal on Scientific Computing, vol. 32, no. 5, pp. 2832-2852, 2010.

[26] M. J. Lai and L. Y. Liu, "A new estimate of restricted isometry constants for sparse solutions," Applied and Computational Harmonic Analysis, vol. 30, no. 3, pp. 402-406, 2011.

[27] H. Ji, C. Liu, Z. Shen, and Y. Xu, "Robust video denoising using Low rank matrix completion," in Proceedings of the 2010 IEEE Computer Society Conference on Computer Vision and Pattern Recognition, CVPR 2010, pp. 1791-1798, June 2010.

[28] A. Beck and M. Teboulle, "A fast iterative shrinkage-thresholding algorithm for linear inverse problems," SIAM Journal on Imaging Sciences, vol. 2, no. 1, pp. 183-202, 2009.

[29] Z. Shen, K.-C. Toh, and S. Yun, "An accelerated proximal gradient algorithm for frame-based image restoration via the balanced approach," SIAM Journal on Imaging Sciences, vol. 4, no. 2, pp. 573-596, 2011.

[30] K. Dabov, A. Foi, and K. Egiazarian, "Video denoising by sparse $3 \mathrm{~d}$ transform-domain collaborative filtering," in Proceedings of the 15th European Signal Processing Conference, vol. 1, p. 7, 2007.

[31] L. Zhang, L. Zhang, X. Mou, and D. Zhang, "Fsim: a feature similarity index for image quality assessment," IEEE Transactions on Image Processing, vol. 20, no. 8, pp. 2378-2386, 2011. 

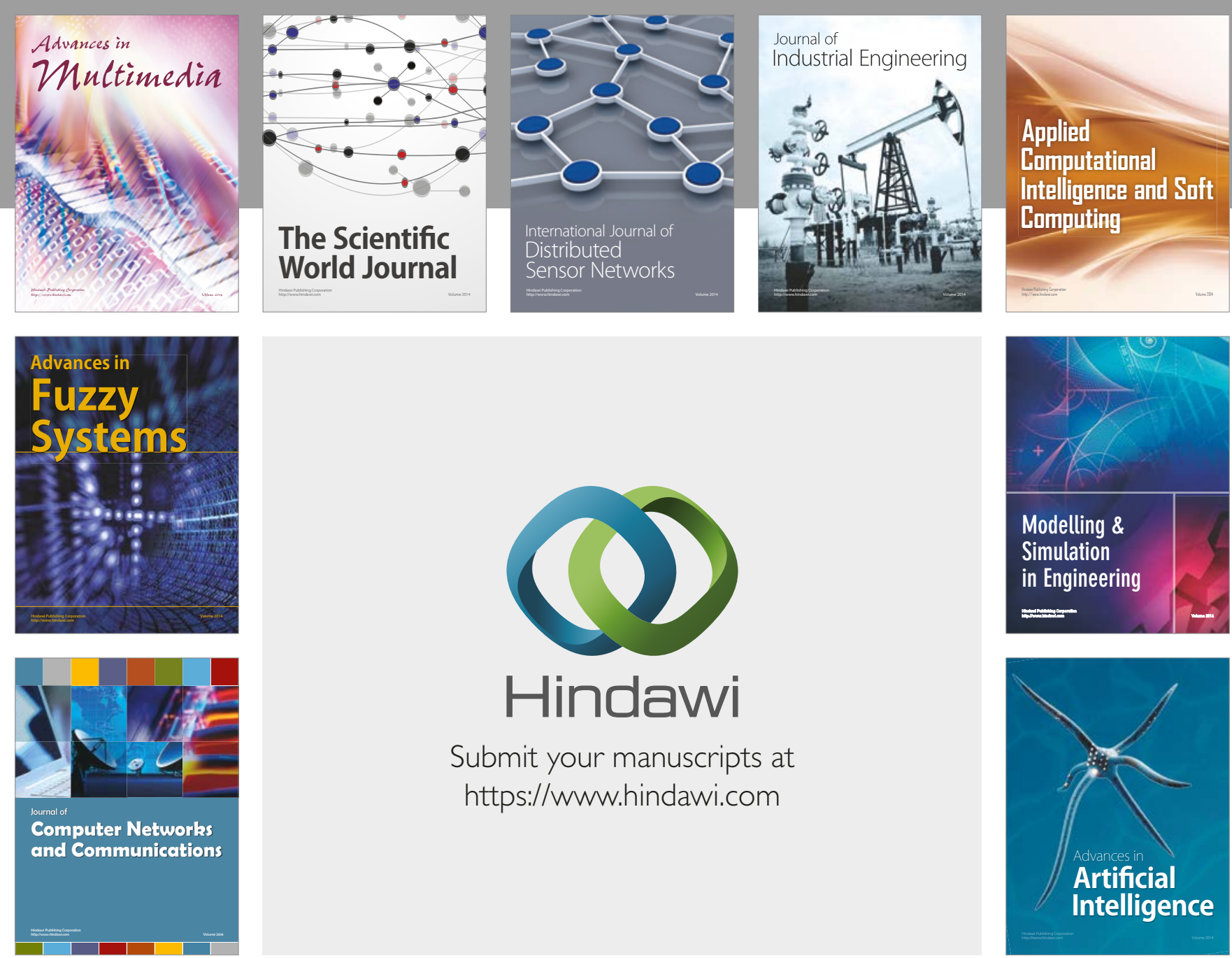

\section{Hindawi}

Submit your manuscripts at

https://www.hindawi.com
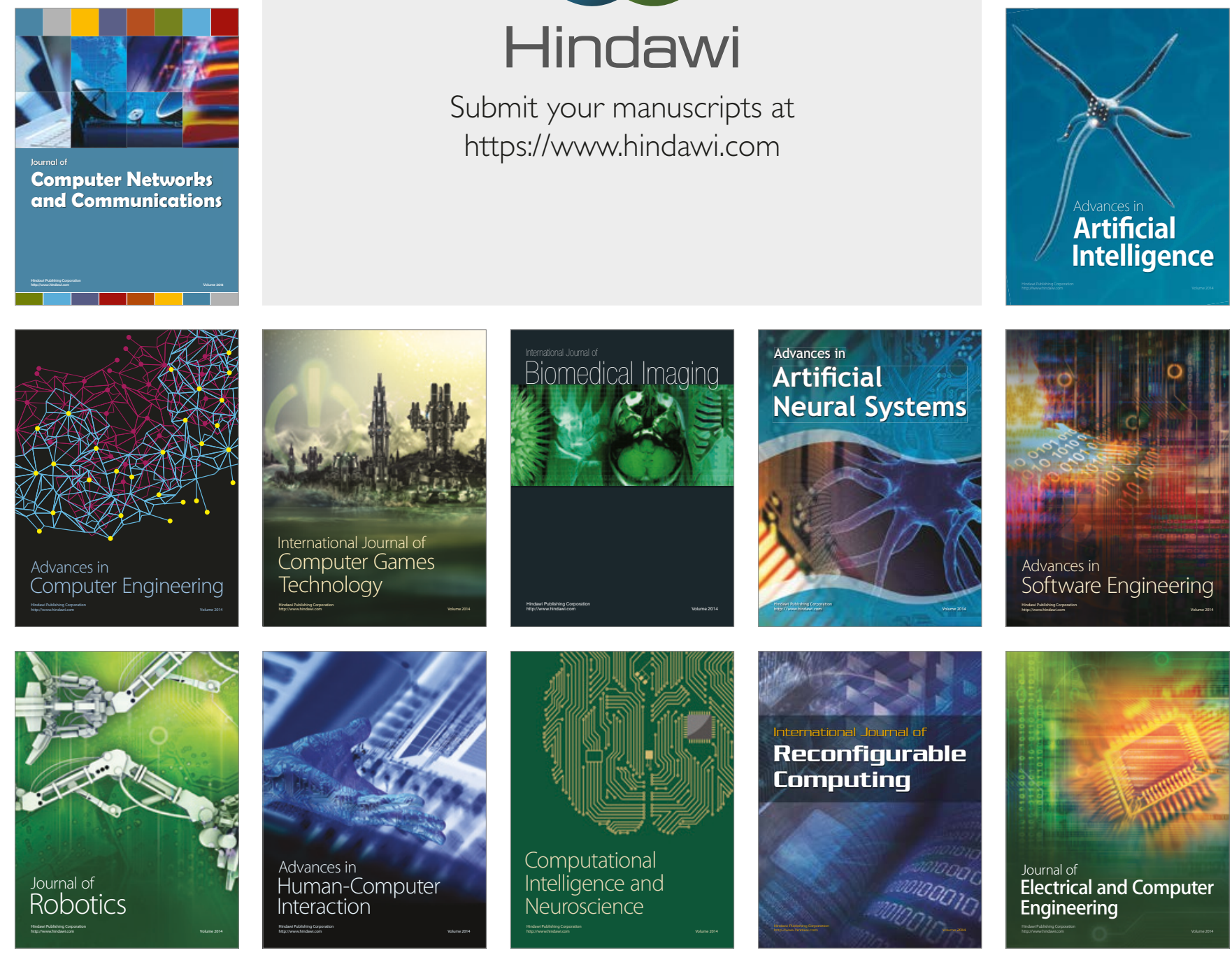